\title{
Linear Combinations and Long Term Trends in the Pulsating DOV PG 1159-035
}

\author{
S. R. Moorhead ${ }^{1}$, M. H. Montgomery ${ }^{1}$, D. E. Winget ${ }^{1}$, and J. Provencal ${ }^{2}$ \\ ${ }^{1}$ Department of Astronomy, University of Texas at Austin \\ ${ }^{2}$ Department of Astronomy and Physics, University of Delaware
}

The star PG 1159-035 ( $\left.\mathrm{T}_{\text {eff }} \approx 140,000 \mathrm{~K}\right)$ is a rapidly evolving pre-white dwarf star that has been a target of the Whole Earth Telescope (WET) for the past 32 years. The nearly continuous observation during each of the eight different WET runs has allowed for the identification of 199 different pulsation modes, and the calculation of period and amplitude change in 27 of those (e.g., see [1], [2]).

Additional observations by the Whole Earth Telescope in 2008, 2014, and 2015 have shown deviations from the predicted evolutionary change of this star and are questioning the existing theory on pulsating DOVs. Specifically, the recent WET observations have yielded two interesting results:

1. Disruption of stable period and amplitude change

2. A linear combination in the Fourier space

Linear combination frequencies are often found in DAV (H-rich envelope) and DBV (He-rich envelope) white dwarf stars and are commonly attributed to nonlinear interactions of the pulsations with the convection zone. DOVs are very hot $\left(\mathrm{T}_{\text {eff }}>100,000 \mathrm{~K}\right)$ and are believed not to possess such surface convection zones. The presence of this nonlinearity is not predicted by current theory and offers new information on the evolutionary changes undergone by a pre-white dwarf star.

It is clear to us now that the star PG 1159-035 has recently experienced significant changes that are yet unexplained by our theoretical understanding of DOV stars. This poster seeks to present and offer initial interpretations of these two exciting phenomena.

Frank N. Bash Symposium 2015

18-20 October

The University of Texas at Austin, USA

\footnotetext{
*Email: sean.moorhead@utexas.edu - This project was supported in part by NSF grant AST-1312983.
} 




Figure 1: Observed period change in the $517.1 \mathrm{~s}$ period from 1983 through 1993: $\frac{d P}{d t} \approx 1.893 \times 10^{-10} \mathrm{~s}$ $\mathrm{s}^{-1}$ and $\frac{d}{d t} \frac{d P}{d t} \approx-2.074 \times 10^{-11} \mathrm{~s} \mathrm{~s}^{-2}$. Beginning in 2002, these rates of period change no longer predict the future values for both the $517.1 \mathrm{~s}$ mode and the $516 \mathrm{~s}$ mode. 2008 - 2015 periods from Period04 [3].

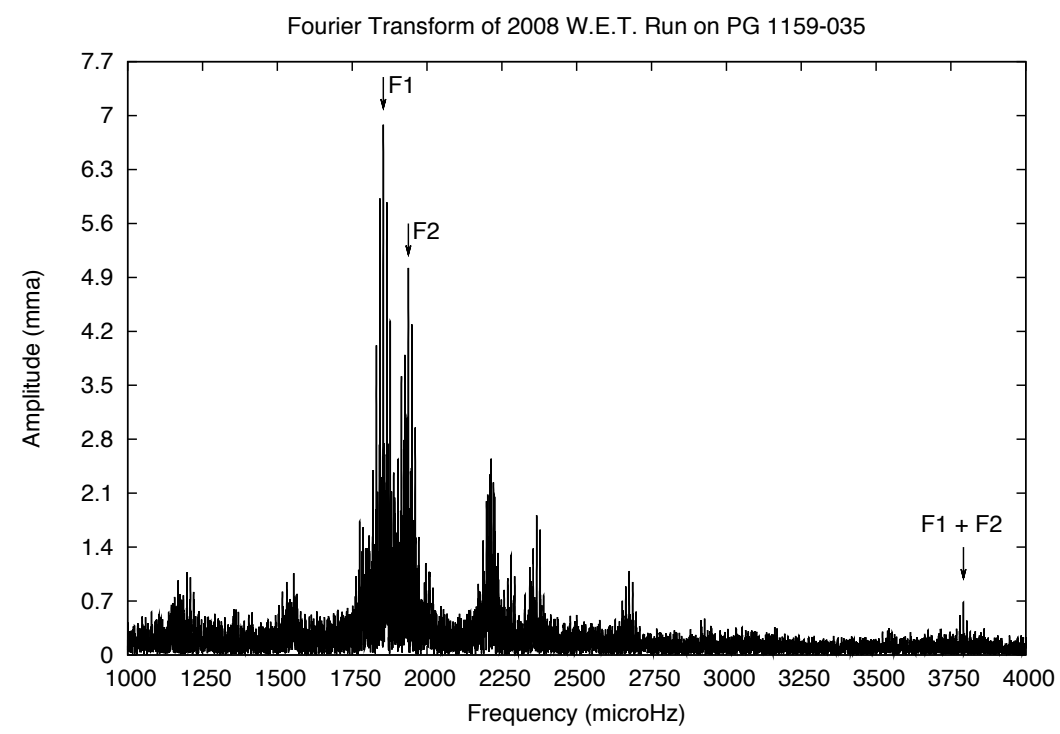

Figure 2: Evidence of a linear combination in the Fourier space. The parent frequencies of $\mathrm{F} 1=1937.599$ $\pm 0.003 \mu \mathrm{Hz}$ and $\mathrm{F} 2=1854.126 \pm 0.005 \mu \mathrm{Hz}$ sum to the combination of $\mathrm{F}_{\text {combo }}=3791.662 \pm 0.041 \mu \mathrm{Hz}$.

\section{References}

[1] J. E. S. Costa, S. O. Kepler, The temporal changes of the pulsational periods of the pre-white dwarf PG 1159-035 2008, A\&A, 489, 1225 [astro-ph/08075137]

[2] J. E. S. Costa, S. O. Kepler, D. E. Winget, et al. The pulsation modes of the pre-white dwarf PG 1159-035 2008, A\&A, 477, 627 [astro-ph/07112244]

[3] P. Lenz \& M. Breger 2005, CoAst, 146, 5 